\title{
Schooling for Multiple Underactuated AUVs
}

\author{
Ji-Hong Li, Bong-Huan Jun, Pan-Mook Lee and Yong-Kon Lim \\ Maritime E Ocean Engineering Research Institute, KORDI, \\ Republic of Korea
}

\section{Introduction}

In the past few decades, autonomous underwater vehicles (AUVs) have been playing one of most important roles in the applications ranging from scientific research, survey to industry and military operations. Today, there is an apparent trend that more and more underwater tasks are carrying out by cooperative operations of multiple AUVs instead of traditional method of using single AUV (Soura \& Pereira, 2002; Edwards et al., 2004; Guo et al., 2004; Watanabe \& Nakamura, 2005; Fiorelli et al., 2006). Multiple AUVs have cost-effective potential. However, a number of research efforts are still remained to be done before this advanced technology can be fully applied in the practice. And one of the efforts is about the efficient schooling scheme for these multiple underwater vehicles.

The history of the formation or cooperative control of multiple agent systems can be traced back to the 1980's. Reynolds (1987) introduced a distributed behavioural model for flocks of birds, herds of land animals, and schools of fishes. This model can be summarized as three heuristic rules: flock centring, collision avoidance and velocity matching. In the formation algorithm (Reynolds, 1987), each dynamic agent was modelled as certain particle system - a simple double-integrator system. This kind of agent model has been inherited in most of the following research works (Leonard \& Fiorelli, 2001; Olfati-Saber \& Murray, 2002, 2003; Fiorelli et al., 2006; Olfati-Saber, 2006; Do, 2007). Besides these works, another type of linear model was used in Smith et al. (2001), and certain nonlinear model was applied for underwater vehicles (Dunbar \& Murray, 2002) and for wheel robots with terminal constraints (Fax \& Murray, 2004). In both of Dunbar \& Murray (2002) and Fax \& Murray (2004), the nonlinear dynamics were all fully actuated.

In this chapter, we consider the schooling problem for multiple underactuated AUVs, where only three control inputs - surge force, stern plane and rudder are available for each vehicle's six degrees of freedom (DOF) motion. For these torpedo-type underwater flying vehicles, since there are non-integrable constraints in the acceleration dynamics, the vehicles do not satisfy Brockett's necessary condition (Brockett et al., 1983), and therefore, could not be asymptotically stabilizable to an equilibrium point using conventional time-invariant continuous feedback laws (Reyhanoglu, 1997; Bacciotti \& Rosier, 2005). Moreover, these vehicles' models are not transformable into a drift-less chained form (Murray \& Sastry, 1993), so the tracking method proposed in Jiang \& Nijmeiner (1999) cannot be directly applicable to these vehicles. Recently, quite a number of research works have been carried out on the tracking of underactuated surface ships (Jiang, 2002; Do et al., 2002a, 2002b, 2004, 2005; Pettersen \& Nijmeijer, 2001; Fredriksen \& Pettersen, 2006). However, the presented

Source: Underwater Vehicles, Book edited by: Alexander V. Inzartsev, ISBN 978-953-7619-49-7, pp. 582, December 2008, I-Tech, Vienna, Austria 
tracking methods were all case-by-case that strongly depended on the ship's specifically simplified dynamics. Therefore, these tracking methods also cannot be directly applicable to the case of underwater vehicles. Since the sway and heave forces are unavailable, the most challenge in the tracking control is how to properly handle the vehicles' sway and heave dynamics in the position tracking. To deal with this problem, in this chapter, we introduce a certain polar coordinates transformation for the vehicle's velocities in the body-fixed frame. Through this coordinates transformation, each vehicle's dynamics can be transformed to a certain two inputs nonlinear strict-feedback form, according to which the proposed schooling scheme is derived.

For the torpedo-type underwater flying vehicles considered in this chapter, the pitch and yaw moments are proportional to the square of the vehicle's forward speed. From this point of view, the pitch and yaw moments are not exactly independent with the surge force. If the vehicle's forward speed is taken small value, then the pitch and yaw moments are also have to take small values, therefore, in this case we cannot fully excite the vehicle's pitch and yaw dynamics. To appropriately taking these three only available control inputs - surge force, pitch and yaw moments as independent ones, the vehicle's forward speed has to be guaranteed to take considerable magnitude. For this reason, in this chapter, firstly we assume that the vehicle's forward speed satisfies the above consideration. And the proposed schooling scheme, which is derived under this assumption, reversely can guarantee the assumption always to be fulfilled under certain initial conditions.

The common method of formation among the schemes presented so far is to apply certain potential function to conduct the agents' group behaviour. The potential function initially used in the robotics for mobile robot's motion planning (Latombe, 1991; Rimon \& Koditschek, 1992), and recently widely applied in the formation of multiple agents systems (Leonard \& Fiorelli, 2001; Olfati-Saber, 2006; Do, 2007). Aforementioned Reynolds's three heuristic rules of flock centring, collision avoidance, and velocity matching, which are also known as cohesion, separation, and alignment, are usually embodied by suitably selected potential functions. In Leonard \& Fiorelli (2001), only 1 time differentiable function was used as potential for group formation, while $p(p \geq 2)$ times differentiable one was applied in Do (2007) and a specific smooth potential was used in Olfati-Saber (2006). In this chapter, general form of smooth potential function is introduced and similar to Olfati-Saber (2006), the potential consists of three different components: one is for the interactions between vehicles, another is for group navigation, and the third is for obstacle avoidance. Unlike aforementioned previous works, in this chapter, the vehicle's orientation is also considered. Therefore, we have to discuss the vehicles orientation matching as well as their velocity matching. Proposed schooling scheme guarantees local minimum of the vehicles formation, and the group's velocity and orientation matching in terms of polar coordinates, while keeping obstacle avoidance.

The remainder of this chapter is organized as follows. In Section 2, the vehicles' kinematics and dynamics in the Cartesian frame are presented. Through certain polar coordinates transformation, the vehicle's model can be transformed to certain two inputs strict-feedback form. Proposed vehicles schooling rules are discussed in Section 3, and corresponding formation control laws are derived in Section 4. To illustrate the effectiveness of proposed schooling scheme, some numerical simulations are carried out and analyzed in Section 5. Finally, a brief summary and some of future works are discussed in Section 6. 


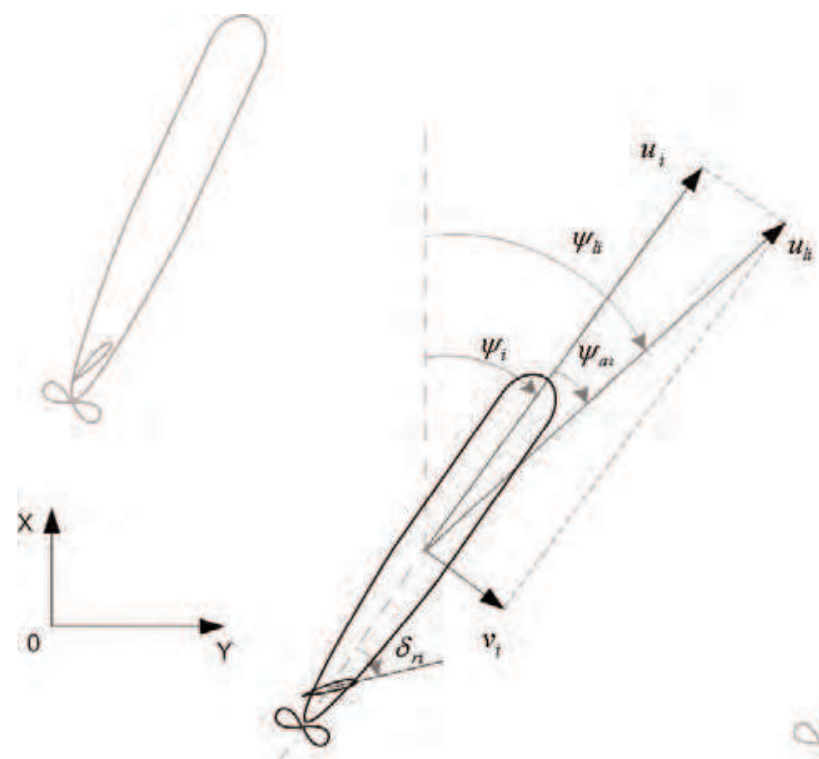

Fig. 1. General framework for an AUV in the horizontal plane.

\section{Problem formulation and preliminaries}

Consider a group of torpedo-type AUVs, where only surge force and yaw moment are available for each vehicle's three DOF horizontal motion ${ }^{1}$, see Fig. 1. To date over 400 true AUVs have been built (Westwood et al., 2007) and most of them such as REMUS AUVs (Prestero, 2001) and HUGIN AUVs (Marthiniussen et al., 2004) have this type of mechanical structure. For this kind of underactuated underwater vehicles, their horizontal kinematics and dynamics can be expressed as following (Fossen, 2002; Li \& Lee, 2008)

$$
\begin{aligned}
& {\left[\begin{array}{c}
\dot{x}_{i} \\
\dot{y}_{i} \\
\dot{\psi}_{i}
\end{array}\right]=\left[\begin{array}{ccc}
\cos \psi_{i} & -\sin \psi_{i} & 0 \\
\sin \psi_{i} & \cos \psi_{i} & 0 \\
0 & 0 & 1
\end{array}\right]\left[\begin{array}{c}
u_{i} \\
v_{i} \\
r_{i}
\end{array}\right],} \\
& {\left[\begin{array}{c}
\dot{u}_{i} \\
\dot{v}_{i} \\
\dot{r}_{i}
\end{array}\right]=\left[\begin{array}{l}
f_{u i}\left(u_{i}, v_{i}, r_{i}\right) \\
f_{v i}\left(u_{i}, v_{i}, r_{i}\right) \\
f_{r i}\left(u_{i}, v_{i}, r_{i}\right)
\end{array}\right]+\left[\begin{array}{cc}
b_{u i} & 0 \\
0 & 0 \\
0 & b_{r i}
\end{array}\right]\left[\begin{array}{l}
\tau_{u i} \\
\tau_{r i}
\end{array}\right],}
\end{aligned}
$$

where $\left(x_{i}, y_{i}\right), i=1, \cdots, n$ denotes the position of the $i$ th vehicle and $\psi_{i}$ is yaw angle, all in the earth-fixed frame; $u_{i}, v_{i}$ and $r_{i}$ denote the velocities each in the surge, sway and yaw

${ }^{1}$ For the convenienceof discussion, in this chapter we only consider the vehicle's three DOF motion in the horizontal plane instead of its full six DOF motion. 
directions in the vehicle's body-fixed frame; $f_{u i}(\cdot), f_{v i}(\cdot), f_{r i}(\cdot) \in C^{1}$ are the vehicle's nonlinear dynamics including hydrodynamic damping, inertia (including added mass terms) and gravitational terms in the surge, sway and yaw directions; surge force $\tau_{u i}$ and yaw moment $\tau_{r i}$ are two available control inputs with nonzero constant gains $b_{u i}$ and $b_{r i}$.

For this torpedo-type underwater flying vehicles, their yaw moments $\tau_{r i}$ is proportional to the square of the forward speed, in other word, $\tau_{r i} \propto u_{i}^{2} \delta_{r i}$ where $\delta_{r i}$ is the vehicle's rudder angle (Fossen, 2002), see Fig. 1. From this point of view, yaw moment $\tau_{r i}$ is not exactly independent of surge force $\tau_{u i}$. Moreover, if the vehicle's forward speed takes value too small, then the yaw moment is also forced to take small value. Therefore, in this case, we could not fully excite the vehicle's yaw dynamics. In order to appropriately taking these two control inputs $\tau_{u i}$ and $\tau_{r i}$ as independent ones, in the remainder of this chapter, we make the following assumption on each vehicle's dynamics.

Assumption 1. For each vehicle in the considering group, its dynamics satisfies the following conditions.

C1. $u_{i} \geq u_{\min }>0$, where $u_{\min }$ is a design parameter.

C2. For bounded $u_{i}$ and $r_{i}, v_{i}$ is also bounded and have $\left|v_{i}\right| \leq v_{\max }$ with $v_{\max }$ a known positive constant.

Remark 1. At first glance, in the above assumption, the condition that $v_{\max }$ is known seems too restrictive in terms of control engineering. However, in the case of underwater vehicles, it becomes very reasonable. For underwater flying vehicles, because of the effect of hydrodynamic damping terms, which is usually proportional to the square of the vehicle's corresponding speed (Fossen, 2002; Newman, 1977), they only have to take limited magnitude of velocities under the limited thrust force. Therefore, in practice, given a torpedo-type flying vehicle, it's maximum forward and sway speeds and yaw angular velocity are all easy to bring out through certain simple experiments such as basin test. From this point of view, it is reasonable for us to design the parameter $u_{\min }$ such that $u_{\min } \geq \rho v_{\max }$. As aforementioned, since the sway force is unavailable, the most difficulty for the control of (1) is how to properly handling the vehicle's sway dynamics. To deal with this problem, firstly we introduce a polar coordinates transformation which is defined in the vehicle's body-fixed frame as following (see Fig. 1)

$$
u_{l i}=\sqrt{u_{i}^{2}+v_{i}^{2}}, \quad \psi_{l i}=\psi_{i}+\psi_{a i},
$$

where $\psi_{a i}=\arctan \left(v_{i} / u_{i}\right)$ is a polar angle and also called as the sideslip angle (Fossen, 2002). Since $u_{i}>0$, it is easy to verify that $\psi_{a i}$ is defined and smooth in the domain $(-0.5 \pi, 0.5 \pi)$. Differentiating the first equation of (2) and further according to the relationships of $u_{i}=u_{l i} \cos \psi_{a i}$ and $v_{i}=u_{l i} \sin \psi_{a i}$, we can get

$$
\dot{u}_{l i}=\dot{u}_{i} \cos \psi_{a i}+\dot{v}_{i} \sin \psi_{a i} .
$$

Using polar coordinates $u_{l i}$ and $\psi_{l i}$, vehicle's kinematics can be rewritten as

$$
\dot{x}_{i}=u_{l i} \cos \psi_{l i}, \quad \dot{y}_{i}=u_{l i} \sin \psi_{l i} .
$$

For the convenience of discussion, in the remainder of this chapter, we will call $u_{l i}$ and $\psi_{l i}$ as the $i$ th vehicle's velocity and heading. 
Combining with (3) and (4), the vehicle's model (1) can be rewritten as following form

$$
\begin{aligned}
& {\left[\begin{array}{c}
\dot{x}_{i} \\
\dot{y}_{i} \\
\dot{\psi}_{l i}
\end{array}\right]=\left[\begin{array}{c}
0 \\
0 \\
\dot{\psi}_{a i}
\end{array}\right]+\left[\begin{array}{cc}
\cos \psi_{l i} & 0 \\
\sin \psi_{l i} & 0 \\
0 & 1
\end{array}\right]\left[\begin{array}{c}
u_{l i} \\
r_{i}
\end{array}\right],} \\
& {\left[\begin{array}{c}
\dot{u}_{l i} \\
\dot{r}_{i}
\end{array}\right]=\left[\begin{array}{c}
f_{u i} \cos \psi_{a i}+f_{v i} \sin \psi_{a i} \\
f_{r i}
\end{array}\right]+\left[\begin{array}{cc}
b_{u i} \cos \psi_{a i} & 0 \\
0 & b_{r i}
\end{array}\right]\left[\begin{array}{c}
\tau_{u i} \\
\tau_{r i}
\end{array}\right] .}
\end{aligned}
$$

Since $\psi_{a i} \in(-0.5 \pi, 0.5 \pi), \cos \psi_{a i} \neq 0$. Therefore, it is easy to see that (5) is in a form where the vehicle's sway dynamics $f_{v i}$ can be properly handled by the surge force $\tau_{u i}$.

\section{Schooling rules}

Consider a group of $n$ underwater vehicles, all of which have the same model as (1). Similar to the Reynolds's (1987) three heuristic rules that are flock centring, collision avoidance and velocity matching, the schooling rules proposed in this chapter can be summarized as: geometry of schooling, group navigation, and obstacle avoidance. As aforementioned, the group behaviour is conducted by suitably selected potential functions.

Unlike the previous works (Leonard \& Fiorelli, 2001; Olfati-Saber, 2006; Do, 2007), in this chapter we consider the following general form of smooth potential function.

Definition 1 (Smooth potential function). A scalar function $f_{p}(\zeta, a, b)$, where $\zeta \in[0,+\infty)$ and $a$ and $b$ are constants with $0<a \leq b$, is called a smooth potential function, if it satisfies the following conditions.

C3. $f_{p}(\zeta, a, b)$ is smooth respect to $\zeta \in[0,+\infty)$ with monotonically decreasing at $\zeta \in[0, a)$ and monotonically increasing at $\zeta \in[a,+\infty)$.

$C 4$. If $a<b$, then $f_{p}(\zeta, a, b)$ has a global minimum of zero at $\zeta=a$ with $\partial f_{p} / \partial \zeta=0$ for $\forall \zeta \geq b$.

C5. If $a=b$, then $f_{p}(\zeta, a, b)=0$ for $\forall \zeta \geq a$.

Remark 2. If $b<+\infty$, then $f_{p}(\zeta, a, b)$ is said to have a finite cut-off (Olfati-Saber, 2006). This kind of feature plays an important role in the group formation (Leonard \& Fiorelli, 2001; Olfati-Saber, 2006; Do, 2007). On the other hand, in Olfati-Saber (2006), the potential function took a finite value when $\zeta \rightarrow 0^{+}$, while it took $+\infty$ in Leonard \& Fiorelli (2001) and Do (2007). From a collision-free point of view, the latter one seems to have its own benefit. However, the finite value case as in Olfati-Saber (2006) may be more natural in practical flocking or schooling.

Remark 3 . There are many functions satisfying C3 C5. For example,

$$
\begin{gathered}
f_{p}(\zeta, a, b)=c \int_{a}^{\zeta}(\tau-a) \rho(\tau / b) d \tau, \\
f_{p}(\zeta, a, b)=c \int_{a}^{\zeta}\left(1 / \tau-a / \tau^{2}\right) \rho(\tau / b) d \tau,
\end{gathered}
$$

where $c>0$ is a constant and $\rho(\cdot)$ is a smooth bump function taken as following 


$$
\rho(\xi)= \begin{cases}1, & \xi \in[0, h) \\ \exp \left[-(\xi-h)^{2} /(\xi-1)^{2}\right], & \xi \in[h, 1] \\ 0, & \text { otherwise }\end{cases}
$$

where $h \in(0,1)$ is a design parameter. It is easy to verify that when $\zeta \rightarrow 0^{+}, f_{p} \rightarrow c a^{2} / 2$ which is a finite value in (6) while $f_{p} \rightarrow+\infty$ in (7). Moreover, in (6), if $b \rightarrow+\infty$, then the potential function becomes a general quadratic form as $c(\zeta-a)^{2} / 2$. For another example of smooth potential and bump functions, refer to Olfati-Saber (2006). Functions (6) and (7) are depicted in Fig. 2.
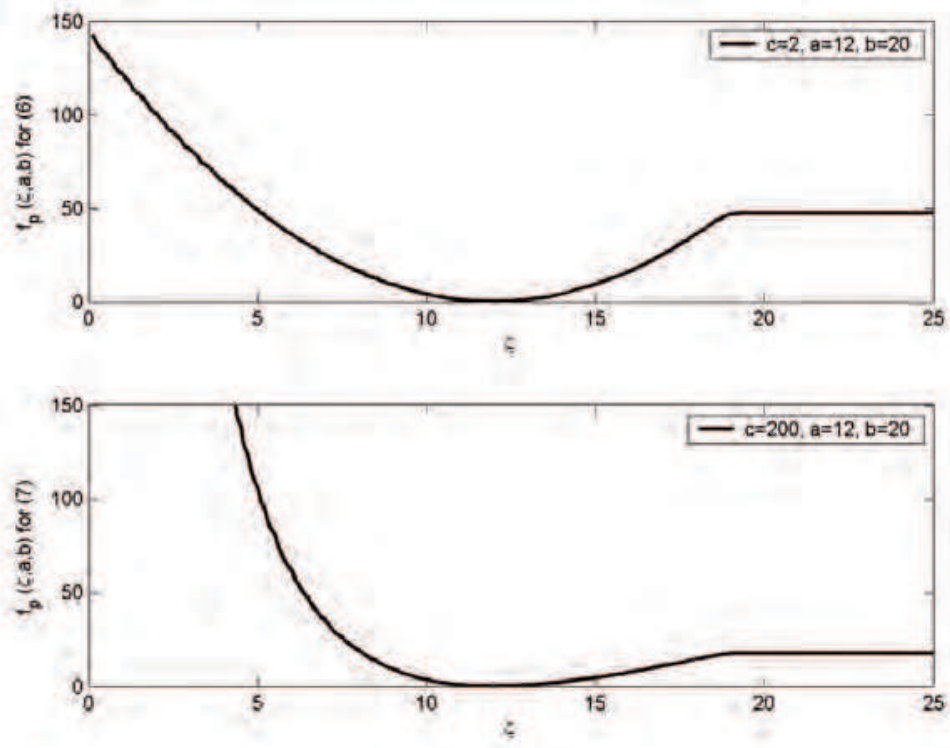

Fig. 2. Smooth potential functions for (6) and (7).

\subsection{Geometry of schooling}

The schooling geometry is constructed according to the following two kinds of potential functions

$$
\begin{gathered}
V_{\alpha}=\sum_{i=1}^{n} \sum_{j \neq i}^{n} f_{p}\left(\left\|q_{j}-q_{i}\right\|, a_{\alpha}, b_{\alpha}\right), \\
V_{\beta}=\sum_{i=1}^{n} \sum_{k=1}^{m} f_{p}\left(\left\|q_{v, k}-q_{i}\right\|, a_{\beta}, b_{\beta}\right),
\end{gathered}
$$

where $q_{i}=\left(x_{i}, y_{i}\right)$ is the $i$ th vehicle's coordinate and $q_{v, k}=\left(x_{v, k}, y_{v, k}\right)$ is the $k$ th virtual vehicle's coordinate with $m$ the number of virtual vehicles needed to conduct the group 
geometry, $a_{\alpha}, b_{\alpha}, a_{\beta}, b_{\beta}>0$ are design parameters with $a_{\alpha} \leq b_{\alpha}$ and $a_{\beta} \leq b_{\beta}$, and $\|\cdot\|$ denotes the vector Euclidean norm.

The potential $V_{\alpha}$ in (9) is presenting the interactions between vehicles. From (9), it is easy to see that the desired geometry of the vehicles group is a certain net of regular triangles with vehicles located at the vertices and all side lengths are equal to $a_{\alpha}$. If any two vehicles are apart from each other more than $b_{\alpha}$, then there is not any cohesion between them. For (9) to be applicable, for each vehicle, all other vehicles' information including position and velocity information should be available. However, in some practical cases, this may not be available. Instead, only the information of the vehicles in its neighbour is available. In this case, the potential function can be chosen as

$$
V_{\alpha}=\sum_{i=1}^{n} \sum_{j \in N\left(q_{i}\right)} f_{p}\left(\left\|q_{j}-q_{i}\right\|, a_{\alpha}, b_{\alpha}\right)
$$

where $N\left(q_{i}\right)$ denotes the neighbour of the $i$ th vehicle and is defined as

$$
N\left(q_{i}\right)=\left\{q_{j}:\left\|q_{j}-q_{i}\right\| \leq b_{\alpha}, j \neq i, j=1, \cdots, n\right\} .
$$

In order to make $V_{\alpha}$ be smooth, in (11), it should be chosen that $a_{\alpha}=b_{\alpha}$.

In (10), the potential function $V_{\beta}$ is for the interactions between vehicles and virtual vehicles. Here the virtual vehicles are introduced to construct the geometry of the vehicles schooling. Moreover, these virtual vehicles are used to guide the group navigation, which will be discussed in details in the next subsection. In other word, these virtual ones lead the group to follow a given desired motion. From this point of view, these virtual vehicles are also called as virtual leaders. Different arrangements of these virtual leaders can lead to different geometry of the schooling, see Fig. 3.

\subsection{Group navigation}

In the literature, group navigation is usually led by certain leaders, which can be some specific actual agents (Guo et al., 2004; Edwards et al., 2004) or some virtual ones (Leonard \& Fiorelli, 2001; Olfati-Saber, 2006). The group movement can be guided through properly designing the reference paths for these virtual leaders. As aforementioned, in this chapter we apply the virtual leader concept to guide the group navigation.

Assumption 2. All virtual leaders move with the same velocity $u_{l v}$ and same heading $\psi_{l v}$. Moreover, we have $u_{l v}>\sqrt{u_{\min }^{2}+v_{\max }^{2}}$.

In fact, these virtual leaders can take any complicated motions, which in turn can lead to various geometry of the schooling. However, the focus of this chapter is taken on that to propose a stable schooling for a group of underactuated underwater vehicles. Therefore, for the convenience of discussion, in this chapter we only consider a simple case where all virtual leaders take the same velocity and heading. Corresponding potential function is chosen as following

$$
V_{g n}=\frac{1}{2} \sum_{i=1}^{n}\left[\gamma_{u}\left(u_{l v}-u_{l i}\right)^{2}+\gamma_{\psi}\left(\psi_{l v}-\psi_{l i}\right)^{2}\right] .
$$

where $\gamma_{u}, \gamma_{\psi}>0$ are weighting factors. 


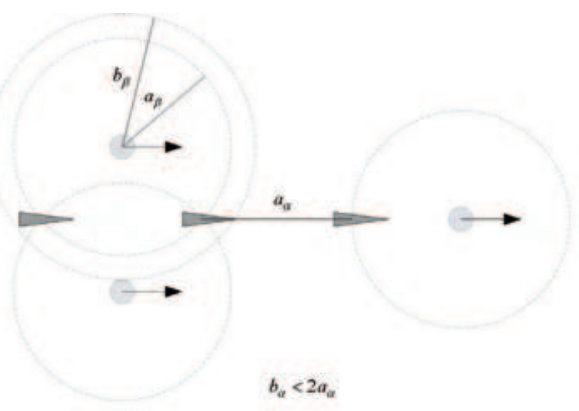

(a) Straight line movement.

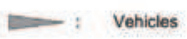

Virtual leaders

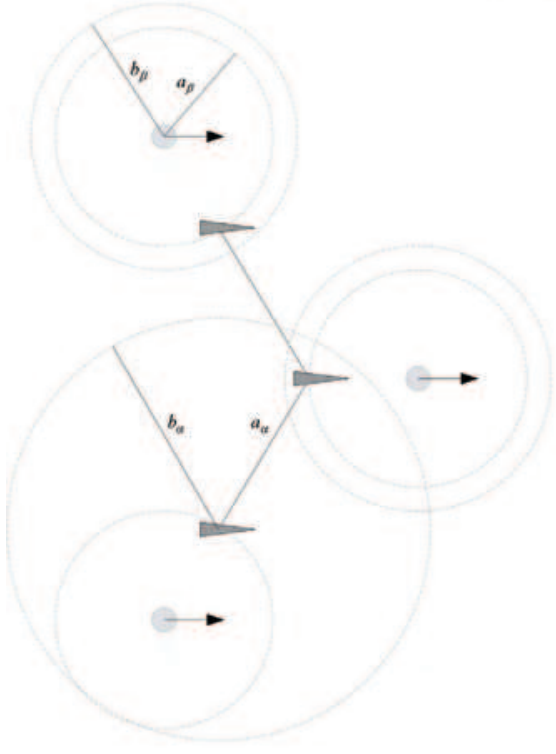

(b) Triangular movement.

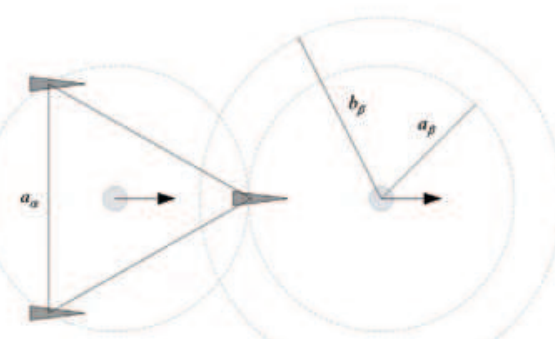

(c) (Equilateral) triangular movement.

Fig. 3. Different arrangements of virtual leaders lead to different geometry of schooling.

From (13), it is easy to see that the purpose of potential $V_{g n}$ is to force the group to keep velocity and heading matching in terms of $u_{l i}$ and $\psi_{l i}$.

\subsection{Obstacle avoidance}

All obstacles considered in this chapter are position fixed. Modelling of these obstacles is as Fig. 4. Inside the circle centred at the $i$ th vehicle $q_{i}$ with radius $b_{\gamma}$, each obstacle block is modelled as the point from which to $q_{i}$ is the shortest. In Fig. 4, B1 and B2 which are two parts of the same obstacle $B$ are considered to be independent and modelled as two different points $q_{i, 2}$ and $q_{i, 3}$. Also, it is notable that the same obstacle such as $B$ in Fig. 4 can be modelled as different points according to different vehicles. 


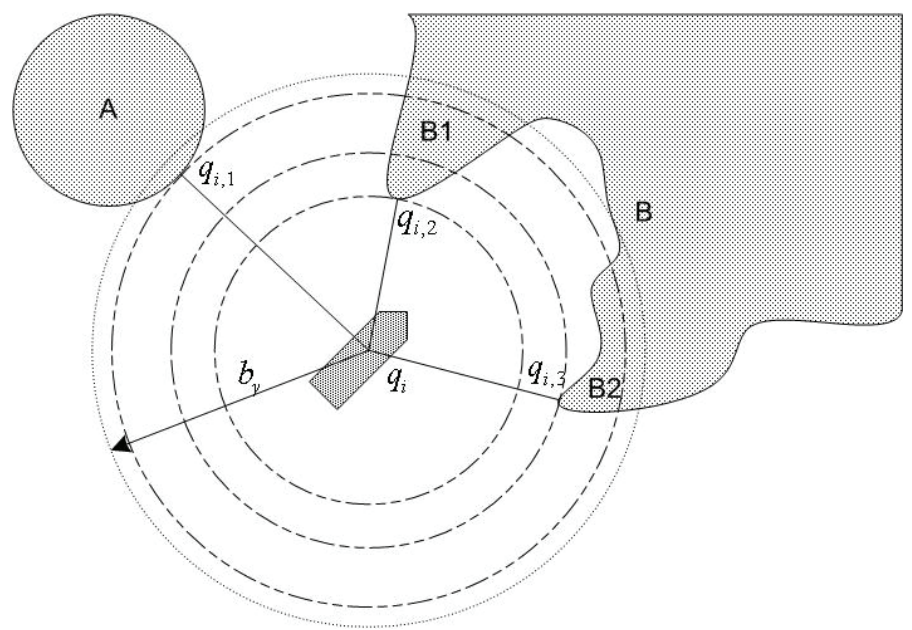

Fig. 4. Obstacle modelling.

Vehicle's obstacle avoidance is also guided by the following potential functions

$$
V_{\gamma}=\sum_{i=1}^{n} \sum_{p \in \Omega\left(q_{i}\right)} f_{p}\left(\left\|q_{i, p}-q_{i}\right\|, a_{\gamma}, b_{\gamma}\right),
$$

where $\Omega\left(q_{i}\right)$ is a subgroup of obstacle points defined as following

$$
\Omega\left(q_{i}\right)=\left\{q_{i, p}:\left\|q_{i, p}-q_{i}\right\| \leq b_{\gamma}, p=1,2, \cdots\right\} .
$$

From obstacle avoidance point of view, there is not any need to have cohesion between vehicles and obstacles. For this reason, in (14), we design the parameters such that $a_{\gamma}=b_{\gamma}$. As aforementioned, in this chapter the group navigation is guided by the virtual leaders. Therefore, the vehicles' obstacle avoidances also have to be strongly related to the motion of virtual leaders. For this reason, we make the following assumption on the motion of the virtual leaders.

Assumption 3. All virtual leaders are designed to satisfying the following conditions.

C6. For any given obstacle, after a certain period of time, all virtual leaders always move away from this obstacle.

C7. After a period of time such that $\min _{k, i}\left\|q_{v, k}-q_{i, p}\right\| \geq b_{\beta}+b_{\gamma}$, if one or more vehicles are still trapped by obstacles, in other word, $\forall k,\left\|q_{v, k}-q_{j}\right\| \geq b_{\beta}$ with $q_{j}$ the trapped vehicle, then all virtual leaders (smoothly) stop movement so that $\dot{q}_{v, k}=0$. Otherwise, C6 still satisfies.

Remark 4. From C7 in Assumption 3, we can see that the obstacle avoidance scenario introduced in this chapter cannot guarantee all vehicles to flee from any given obstacles. Instead, we only try to guarantee the vehicles to collision-free with obstacles. For example, as in $C 7$, if one or more vehicles are trapped by obstacles, then we force these vehicle to stop movement so as to avoid collision with obstacles. In fact, obstacle avoidance is still being a complicated and open issue in the practical robotics. How to guide the group to move through the obstacles is out of the scope of this chapter. 


\section{Formation control design}

In this section, we will propose a formation control algorithm that can guarantee the stable schooling for multiple underactuated underwater vehicles whose models can be expressed as (1) or (5) with Assumption 1. It is easy to see that (5) is in a second-order nonlinear strictfeedback form, and we will solve the schooling problem using general backstepping method (Krstic et al., 1995).

Step 1. As aforementioned, the vehicles' schooling is conducted by the various potential functions introduced in the previous section. For this reason, in this step, we consider the following Lyapunov function candidate

$$
V_{1}=\gamma_{\alpha} V_{\alpha}+\gamma_{\beta} V_{\beta}+\gamma_{\gamma} V_{\gamma}+V_{g n},
$$

where $\gamma_{\alpha}, \gamma_{\beta}, \gamma_{\gamma}>0$ are weighting factors.

Differentiating (16) and substituting (9), (10), (13), and (14) into it, get

$$
\begin{gathered}
\dot{V}_{1}=\gamma_{\alpha} \sum_{i=1}^{n} \sum_{j \neq i}^{n} \frac{\partial f_{p}\left(\left\|q_{j}-q_{i}\right\|, a_{\alpha}, b_{\alpha}\right)}{\partial\left(\left\|q_{j}-q_{i}\right\|\right)}\left[\frac{x_{j}-x_{i}}{\left\|q_{j}-q_{i}\right\|}\left(\dot{x}_{j}-\dot{x}_{i}\right)+\frac{y_{j}-y_{i}}{\left\|q_{j}-q_{i}\right\|}\left(\dot{y}_{j}-\dot{y}_{i}\right)\right] \\
+\gamma_{\beta} \sum_{i=1}^{n} \sum_{k=1}^{m} \frac{\partial f_{p}\left(\left\|q_{v, k}-q_{i}\right\|, a_{\beta}, b_{\beta}\right)}{\partial\left(\left\|q_{v, k}-q_{i}\right\|\right)}\left[\frac{x_{v, k}-x_{i}}{\left\|q_{v, k}-q_{i}\right\|}\left(\dot{x}_{v, k}-\dot{x}_{i}\right)+\frac{y_{v, k}-y_{i}}{\left\|q_{v, k}-q_{i}\right\|}\left(\dot{y}_{v, k}-\dot{y}_{i}\right)\right] \\
+\gamma_{\gamma} \sum_{i=1}^{n} \sum_{p \in \Omega\left(q_{i}\right)} \frac{\partial f_{p}\left(\left\|q_{i, p}-q_{i}\right\|, a_{\gamma}, b_{\gamma}\right)}{\partial\left(\left\|q_{i, p}-q_{i}\right\|\right)}\left[\frac{x_{i, p}-x_{i}}{\left\|q_{i, p}-q_{i}\right\|}\left(\dot{x}_{i, p}-\dot{x}_{i}\right)+\frac{y_{i, p}-y_{i}}{\left\|q_{i, p}-q_{i}\right\|}\left(\dot{y}_{i, p}-\dot{y}_{i}\right)\right] \\
+\sum_{i=1}^{n}\left[\gamma_{u}\left(u_{l v}-u_{l i}\right)\left(\dot{u}_{l v}-\dot{u}_{l i}\right)+\gamma_{\psi}\left(\psi_{l v}-\psi_{l i}\right)\left(\dot{\psi}_{l v}-\dot{\psi}_{l i}\right)\right] .
\end{gathered}
$$

For expression convenience, we apply the following simplifications in the remainder of this chapter that $\partial f_{p}(\zeta, a, b)=\partial f_{p}(\zeta, a, b) / \partial \zeta, \xi_{j i}=\xi_{j}-\xi_{i}, \xi_{v k i}=\xi_{v, k}-\xi_{i}$, and $\xi_{i p i}=\xi_{i, p}-\xi_{i}$ with $\xi=q, x, y$, and $u_{l v i}=u_{l v}-u_{l i}, \psi_{l v i}=\psi_{l v}-\psi_{l i}$. As aforementioned, all virtual leaders have the same velocity and heading with the kinematics of $\dot{x}_{v, k}=u_{l v} \cos \psi_{l v}$ and $\dot{y}_{v, k}=u_{l v} \sin \psi_{l v}$. Substituting (5) into (17) and further expanding it, we can get

$$
\begin{gathered}
\dot{V}_{1}=\gamma_{\alpha} \sum_{i=1}^{n} \sum_{j \neq i}^{n} \partial f_{p}\left(\left\|q_{j i}\right\|, a_{\alpha}, b_{\alpha}\right)\left[\frac{x_{j i}}{\left\|q_{j i}\right\|}\left[\left(\dot{x}_{j}-u_{l v} \cos \psi_{l v}\right)+\left(u_{l v} \cos \psi_{l v}-\dot{x}_{i}\right)\right]\right. \\
\left.+\frac{y_{j i}}{\left\|q_{j i}\right\|}\left[\left(\dot{y}_{j}-u_{l v} \sin \psi_{l v}\right)+\left(u_{l v} \sin \psi_{l v}-\dot{y}_{i}\right)\right]\right] \\
+\gamma_{\beta} \sum_{i=1}^{n} \sum_{k=1}^{m} \partial f_{p}\left(\left\|q_{v k i}\right\|, a_{\beta}, b_{\beta}\right)\left[\frac{x_{v k i}}{\left\|q_{v k i}\right\|}\left(u_{l v} \cos \psi_{l v}-\dot{x}_{i}\right)+\frac{y_{v k i}}{\left\|q_{v k i}\right\|}\left(u_{l v} \sin \psi_{l v}-\dot{y}_{i}\right)\right]
\end{gathered}
$$




$$
\begin{aligned}
& -\gamma_{\gamma} \sum_{i=1}^{n} \sum_{p \in \Omega\left(q_{i}\right)} \partial f_{p}\left(\left\|q_{i p i}\right\|, a_{\gamma}, b_{\gamma}\right)\left(\frac{x_{i p i}}{\left\|q_{i p i}\right\|} \dot{x}_{i}+\frac{y_{i p i}}{\left\|q_{i p i}\right\|} \dot{y}_{i}\right) \\
& +\sum_{i=1}^{n}\left[\gamma_{u} u_{l v i}\left(\dot{u}_{l v}-\dot{u}_{l i}\right)+\gamma_{\psi} \psi_{l v i}\left(\dot{\psi}_{l v}-\dot{\psi}_{l i}\right)\right] \\
& =2 \gamma_{\alpha} \sum_{i=1}^{n} \sum_{j \neq i}^{n} \partial f_{p}\left(\left\|q_{j i}\right\|, a_{\alpha}, b_{\alpha}\right)\left[\frac{x_{j i}}{\left\|q_{j i}\right\|}\left(u_{l v} \cos \psi_{l v}-\dot{x}_{i}\right)+\frac{y_{j i}}{\left\|q_{j i}\right\|}\left(u_{l v} \sin \psi_{l v}-\dot{y}_{i}\right)\right] \\
& +\gamma_{\beta} \sum_{i=1}^{n} \sum_{k=1}^{m} \partial f_{p}\left(\left\|q_{v k i}\right\|, a_{\beta}, b_{\beta}\right)\left[\frac{x_{v k i}}{\left\|q_{v k i}\right\|}\left(u_{l v} \cos \psi_{l v}-\dot{x}_{i}\right)+\frac{y_{v k i}}{\left\|q_{v k i}\right\|}\left(u_{l v} \sin \psi_{l v}-\dot{y}_{i}\right)\right] \\
& +\gamma_{\gamma} \sum_{i=1}^{n} \sum_{p \in \Omega\left(q_{i}\right)} \partial f_{p}\left(\left\|q_{i p i}\right\|, a_{\gamma}, b_{\gamma}\right)\left[\frac{x_{i p i}}{\left\|q_{i p i}\right\|}\left(u_{l v} \cos \psi_{l v}-\dot{x}_{i}\right)+\frac{y_{i p i}}{\left\|q_{i p i}\right\|}\left(u_{l v} \sin \psi_{l v}-\dot{y}_{i}\right)\right] \\
& -\gamma_{\gamma} \sum_{i=1}^{n} \sum_{p \in \Omega\left(q_{i}\right)} \partial f_{p}\left(\left\|q_{i p i}\right\|, a_{\gamma}, b_{\gamma}\right)\left(\frac{x_{i p i}}{\left\|q_{i p i}\right\|} u_{l v} \cos \psi_{l v}+\frac{y_{i p i}}{\left\|q_{i p i}\right\|} u_{l v} \sin \psi_{l v}\right) \\
& +\sum_{i=1}^{n}\left[\gamma_{u} u_{l v i}\left(\dot{u}_{l v}-\dot{u}_{l i}\right)+\gamma_{\psi} \psi_{l v i}\left(\dot{\psi}_{l v}-\dot{\psi}_{l i}\right)\right] \\
& =\sum_{i=1}^{n}\left[\Lambda_{x i}\left(u_{l v} \cos \psi_{l v}-u_{l i} \cos \psi_{l i}\right)+\Lambda_{y i}\left(u_{l v} \sin \psi_{l v}-u_{l i} \sin \psi_{l i}\right)+\Lambda_{o}\right. \\
& \left.+\gamma_{u} u_{l v i}\left(\dot{u}_{l v}-\dot{u}_{l i}\right)+\gamma_{\psi} \psi_{l v i}\left(\dot{\psi}_{l v}-\dot{\psi}_{l i}\right)\right\rfloor \\
& =\sum_{i=1}^{n}\left[\left(\Lambda_{x i} \cos \psi_{l i}+\Lambda_{y i} \sin \psi_{l i}\right) u_{l v i}+2 u_{l v}\left(\Lambda_{y i} \cos \frac{\psi_{l v}+\psi_{l i}}{2}-\Lambda_{x i} \sin \frac{\psi_{l v}+\psi_{l i}}{2}\right) \sin \frac{\psi_{l v i}}{2}\right. \\
& \left.+\Lambda_{o}+\gamma_{u} u_{l v i}\left(\dot{u}_{l v}-f_{u i} \cos \psi_{a i}-f_{v i} \sin \psi_{a i}-b_{u i} \cos \psi_{a i} \cdot \tau_{u i}\right)+\gamma_{\psi} \psi_{l v i}\left(\dot{\psi}_{l v}-\dot{\psi}_{a i}-\alpha_{r i}+e_{r i}\right)\right]
\end{aligned}
$$

where $e_{r i}=\alpha_{r i}-r_{i}$ with $\alpha_{r i}$ a stabilizing function (Krstic et al., 1995) for virtual input $r_{i}$, $\Lambda_{x i}$ and $\Lambda_{o}$ are defined as following

$$
\begin{gathered}
\Lambda_{x i}=2 \gamma_{\alpha} \sum_{j \neq i}^{n} \partial f_{p}\left(\left\|q_{j i}\right\|, a_{\alpha}, b_{\alpha}\right) \frac{x_{j i}}{\left\|q_{j i}\right\|}+\gamma_{\beta} \sum_{k=1}^{m} \partial f_{p}\left(\left\|q_{v k i}\right\|, a_{\beta}, b_{\beta}\right) \frac{x_{v k i}}{\left\|q_{v k i}\right\|} \\
+\gamma_{\gamma} \sum_{p \in \Omega(q i)} \partial f_{p}\left(\left\|q_{i p i}\right\|, a_{\gamma}, b_{\gamma}\right) \frac{x_{i p i}}{\left\|q_{i p i}\right\|}, \\
\Lambda_{o}=-\gamma_{\gamma} \sum_{p \in \Omega\left(q_{i}\right)} \partial f_{p}\left(\left\|q_{i p i}\right\|, a_{\gamma}, b_{\gamma}\right)\left(\frac{x_{i p i}}{\left\|q_{i p i}\right\|} u_{l v} \cos \psi_{l v}+\frac{y_{i p i}}{\left\|q_{i p i}\right\|} u_{l v} \sin \psi_{l v}\right),
\end{gathered}
$$

and $\Lambda_{y i}$ is defined to have the same form with $\Lambda_{x i}$ with only $x$ displaced by $y$. 
According to (18), in this step we choose the control laws as following

$$
\begin{aligned}
& \tau_{u i}=b_{u i}^{-1} \sec \psi_{a i}\left[\dot{u}_{l v}-f_{u i} \cos \psi_{a i}-f_{v i} \sin \psi_{a i}+\gamma_{u}^{-1}\left(k_{u i} u_{l v i}+\Lambda_{x i} \cos \psi_{l i}+\Lambda_{y i} \sin \psi_{l i}\right)\right], \\
& \alpha_{r i}=\dot{\psi}_{l v}-\dot{\psi}_{a i}+\gamma_{\psi}^{-1}\left[k_{\psi i} \psi_{l v i}+u_{l v}\left(\Lambda_{y i} \cos \frac{\psi_{l v}+\psi_{l i}}{2}-\Lambda_{x i} \sin \frac{\psi_{l v}+\psi_{l i}}{2}\right) \frac{\sin \left(\psi_{l v i} / 2\right)}{\psi_{l v i} / 2}\right],
\end{aligned}
$$

where $k_{u i}, k_{\psi i}>0$ are design parameters.

Remark 5. According to (2), we can see that $\dot{\psi}_{a i}$ contains the acceleration terms $\dot{u}$ and $\dot{v}$. However, since $f_{u i}(\cdot), f_{v i}(\cdot), f_{r i}(\cdot) \in C^{2}$, it is not difficult to verify that the surge force control law (21) is differentiable, and this means that $\dot{u}$ and $\dot{v}$ are also differentiable. Consequently, we can conclude that $\dot{\psi}_{a i}$ is also differentiable and so is $\alpha_{r i}$.

Substituting (21) and (22) into (18), get

$$
\dot{V}_{1}=\sum_{i=1}^{n}\left(-k_{u i} u_{l v i}^{2}-k_{\psi i} \psi_{l v i}^{2}+\gamma_{\psi} e_{r i} \psi_{l v i}+\Lambda_{o}\right) .
$$

Step 2. Rewrite the final equation of (5) as following

$$
\dot{e}_{r i}=\dot{\alpha}_{r i}-f_{r i}-b_{r i} \tau_{r i} .
$$

Now, consider the following Lyapunov function candidate

$$
V_{2}=V_{1}+\frac{1}{2} \sum_{i=1}^{n} e_{r i}^{2}
$$

Differentiating (25) and substituting (23) (24) into it, finally we can have

$$
\dot{V}_{2}=\sum_{i=1}^{n}\left[-k_{u i} u_{l v i}^{2}-k_{\psi i} \psi_{l v i}^{2}+\gamma_{\psi} e_{r i} \psi_{l v i}+e_{r i}\left(\dot{\alpha}_{r i}-f_{r i}-b_{r i} \tau_{r i}\right)+\Lambda_{o}\right] .
$$

According to (26), the control law for $\tau_{r i}$ is chosen as

$$
\tau_{r i}=b_{r i}^{-1}\left(k_{r i} e_{r i}+\dot{\alpha}_{r i}-f_{r i}+\gamma_{\psi} \psi_{l v i}\right),
$$

where $k_{r i}>0$ is a design parameter. Substituting (27) into (26), finally we can have

$$
\dot{V}_{2}=\sum_{i=1}^{n}\left(-k_{u i} u_{l v i}^{2}-k_{\psi i} \psi_{l v i}^{2}-k_{r i} e_{r i}^{2}+\Lambda_{o}\right) .
$$

According to Assumption 3, after a certain period of time such that $\min _{k, i}\left\|q_{v, k}-q_{i, p}\right\| \geq b_{\beta}+b_{\gamma}$, if all vehicles are still following the virtual leaders (this means $\left\|q_{k v i}\right\| \leq b_{\beta}$ ), then $\dot{f}_{p}\left(\left\|q_{i p i}\right\|, a_{\gamma}, b_{\gamma}\right)=0, \forall i=1, \cdots, n$ and $\forall p \in \Omega\left(q_{i}\right)$. Otherwise, we have $u_{l v}=0$. In both cases, it is easy to verify that $\Lambda_{o}=0$. Consequently, after a certain period of time, we always have $\dot{V}_{2} \leq 0$, and $\dot{V}_{2}=0$ if and only if $u_{l v i}=\psi_{l v i}=e_{r i}=0, \forall i=1, \cdots, n$.

Theorem. Consider the schooling for multiple underactuated underwater vehicles whose kinematics and dynamics can be expressed as (1) with Assumption 1 3. If we choose the formation control laws as (21) and (27), then the schooling asymptotically converges to a 
certain local minimum and all vehicles included in the group asymptotically move with the same velocity and heading while keeping obstacle avoidance.

Remark 6. Consider the case where one or more vehicles are trapped by obstacles. If we design $V_{\beta}$ to take finite cut-off $\left(b_{\beta}<+\infty\right)$, which is different from Olfati-Saber (2006) and Do (2007), then after $\left\|q_{v k j}\right\| \geq b_{\beta}$ with $q_{j}$ trapped vehicles, we have $\dot{f}_{p}\left(\left\|q_{v k j}\right\|, a_{\beta}, b_{\beta}\right)=0$. This means that after a certain period of time, only two kinds of potentials $V_{\alpha}$ and $V_{\gamma}$ are remained to restrict the behaviour of the trapped vehicles. Therefore, we can guarantee the trapped vehicles to collision-free with obstacles even without C7 in Assumption 3.

Remark 7. So far, we derive the schooling algorithms under the condition $u_{i} \geq u_{\min } \geq \rho v_{\max }$ as in Assumption 1. In fact, substituting (21) into (3), we have

$$
\dot{u}_{l v i}=-\gamma_{u}^{-1} k_{u i} u_{l v i}-\gamma_{u}^{-1}\left(\Lambda_{x i} \cos \psi_{l i}+\Lambda_{y i} \sin \psi_{l i}\right) .
$$

On the other hand, if we take the smooth potential function as the form of (6), then it is not difficult to verify that $\partial f_{p}$ is bounded. Further according to (19), we can see that $\Lambda_{x i}$ and $\Lambda_{y i}$ are also bounded. Therefore, through proper selection of weighting factors $\gamma_{\alpha}, \gamma_{\beta}$ and $\gamma_{\gamma}$, we can make $\Lambda_{x i} \cos \psi_{l i}+\Lambda_{y i} \sin \psi_{l i}$ arbitrarily small. Consequently, further through proper selection of $k_{u i}$, it is easy to get

$$
\left|u_{l v i}(t)\right| \leq\left|u_{l v i}(0)\right| .
$$

Therefore, if we design the virtual leaders' motions such that $u_{l v} \geq \sqrt{v_{\max }^{2}+u_{\min }^{2}}+U$ with $U>0$ a design parameter, then it is not difficult to verify that $\forall t \geq 0$, have $u_{i}(t) \geq u_{\text {min }}$ under the initial condition $\left|u_{l v i}(0)\right| \leq U$. In other word, assumption of $u_{i}(t) \geq u_{\min }$ always can be guaranteed in practice under suitable selection of initial conditions. Meanwhile, this means that the proposed formation control method only guarantees local stability of the schooling. Here it is notable that $\left|u_{l v i}(0)\right| \leq U$ is a certain sufficient condition for $u(t) \geq u_{\min }$, not a necessary one.

Remark 8. Since polar coordinate transformation does not satisfy to be a diffeomorphism, usually $u_{l v i}, \psi_{l v i} \rightarrow 0$ does not guarantee the same convergence properties of $u_{i} \rightarrow u_{v}, v_{i} \rightarrow$ $v_{v}$ and $r_{i} \rightarrow r_{v}$, where $u_{v}, v_{v}, r_{v}$ are the virtual leader's velocity components. This may be one of disadvantages to applying polar coordinate transformation. However, in practical applications, it is much more difficult to design the motion of virtual leaders in the Cartesian frame. For example, for given $u_{v}$ and $r_{v}$, because of high nonlinearity, it is difficult to directly calculate $v_{v}$ through $\dot{v}_{v}=f_{v}\left(u_{v}, v_{v}, r_{v}\right)$. On the contrary, in the corresponding polar frame, we can easily bring out the reference paths for virtual leaders from given $u_{l v}$ and $\psi_{l v}$.

\section{Simulation studies}

In this section, we carry out some simulation studies to illustrate the effectiveness of proposed vehicles schooling scheme. In the simulation, the vehicles group consists of three vehicles, and each of them is modelled as the six DOF nonlinear dynamics of ISiMI AUV (Lee et al., 2003), which has the similar mechanical structure as REMUS AUV (Prestero, 2001). For this ISiMI AUV, we use the saturation conditions as $\left|\tau_{u i}\right| \leq 50 N$ and $\left|\delta_{r i}\right| \leq \pi / 6 \mathrm{rad}$ in the simulation. And all virtual leaders are assumed move with the same velocity and heading as $u_{l v}=1.54 \mathrm{~m} / \mathrm{s}, \psi_{l v}=0 \mathrm{rad}$. 


\subsection{Straight line schooling}

In this case, we can choose the three virtual leaders to locate at the vertices of a certain isosceles triangle as in Fig. 3 (a) with the initial positions as $q_{v 1}(0)=(6,10), q_{v 2}(0)=(6,26)$ and $q_{v 3}(0)=(34,18)$. Three vehicles' initial conditions are as: $q_{1}(0)=(0,24), q_{2}(0)=(12,12)$, $q_{3}(0)=(28,16), \psi_{1}(0)=0.1 \mathrm{rad}, \psi_{2}(0)=0.05 \mathrm{rad}, \psi_{3}(0)=0.15 \mathrm{rad}$, and $u_{1}(0)=u_{2}(0)=u_{3}(0)=0.5$ $\mathrm{m} / \mathrm{s}$ with all other variables taking zero values. Other design parameters are taken as: $k_{u i}=$ $8, k_{\psi i}=3, k_{r i}=12, i=1, \cdots, 3$ and $\gamma_{\alpha}=0.3, \gamma_{\beta}=0.5, \gamma_{u}=100, \gamma_{\psi}=0.2$.

For potential functions $V_{\alpha}$ and $V_{\beta}$, both of them take the form as (6) and corresponding parameters are chosen as: $a_{\alpha}=12, b_{\alpha}=20, a_{\beta}=10, b_{\beta}=20, c_{\alpha}=2, c_{\beta}=3$ and $h=0.9$.

Simulation results are shown in Fig. 5 and 6. Fig. 5 shows the vehicles group schooling in its straight line movement. From Fig. 6, we can see that there is not any collision between vehicles in the schooling.

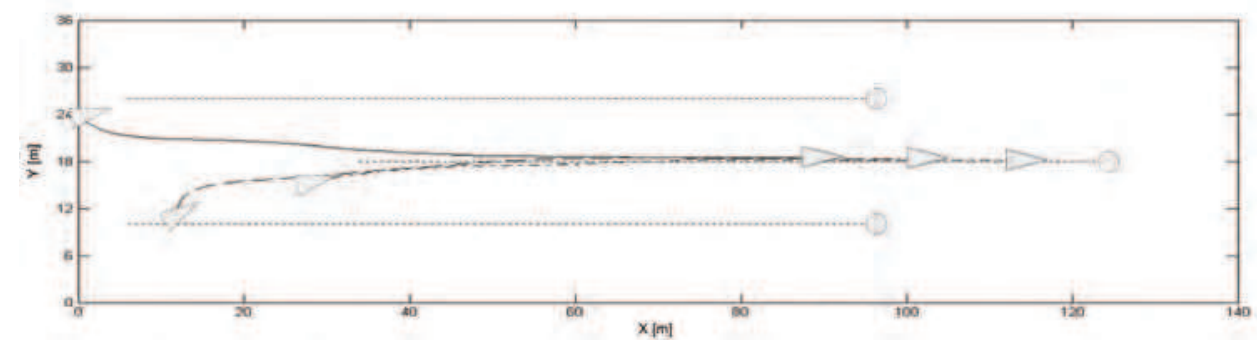

Fig. 5. Schooling of the vehicles in a straight-line movement.

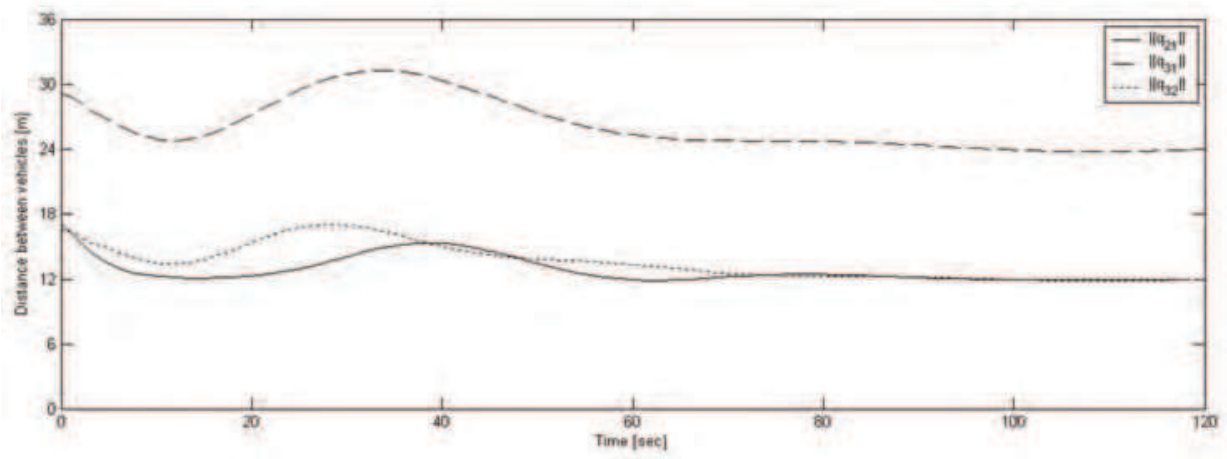

Fig. 6. Schooling geometry for a straight-line movement.

\subsection{Triangular schooling}

For triangular schooling, we locate the three virtual leaders as in Fig. 3 (b) with the initial positions taken as $q_{v 1}(0)=(0,40), q_{v 2}(0)=(0,8)$ and $q_{v 3}(0)=(20,24)$. Three vehicles' initial conditions are chosen as $q_{1}(0)=(9,39), q_{2}(0)=(3,10), q_{3}(0)=(10,22), \psi_{1}(0)=0.1 \mathrm{rad}, \psi_{2}(0)=$ 
$0.05 \mathrm{rad}, \psi_{3}(0)=0.15 \mathrm{rad}$, and $u_{1}(0)=u_{2}(0)=u_{3}(0)=0.5 \mathrm{~m} / \mathrm{s}$ with all other variables taking zero values. Other design parameters are taken as $k_{u i}=12, k_{\psi i}=8, k_{r i}=12, i=1, \cdots, 3$ and $\gamma_{\alpha}=0.3, \gamma_{\beta}=0.5, \gamma_{u}=60, \gamma_{\psi}=0.3$.

Same as in the straight line case, both of $V_{\alpha}$ and $V_{\beta}$ take the form as (6) with the parameters chosen as $a_{\alpha}=12, b_{\alpha}=15, a_{\beta}=8, b_{\beta}=20, c_{\alpha}=2, c_{\beta}=3$ and $h=0.9$.

Corresponding simulation results are presented in Fig. 7 and 8 . The vehicles schooling in the triangular movement is shown in Fig. 7 with no collision between any of two vehicles (see Fig. 8).

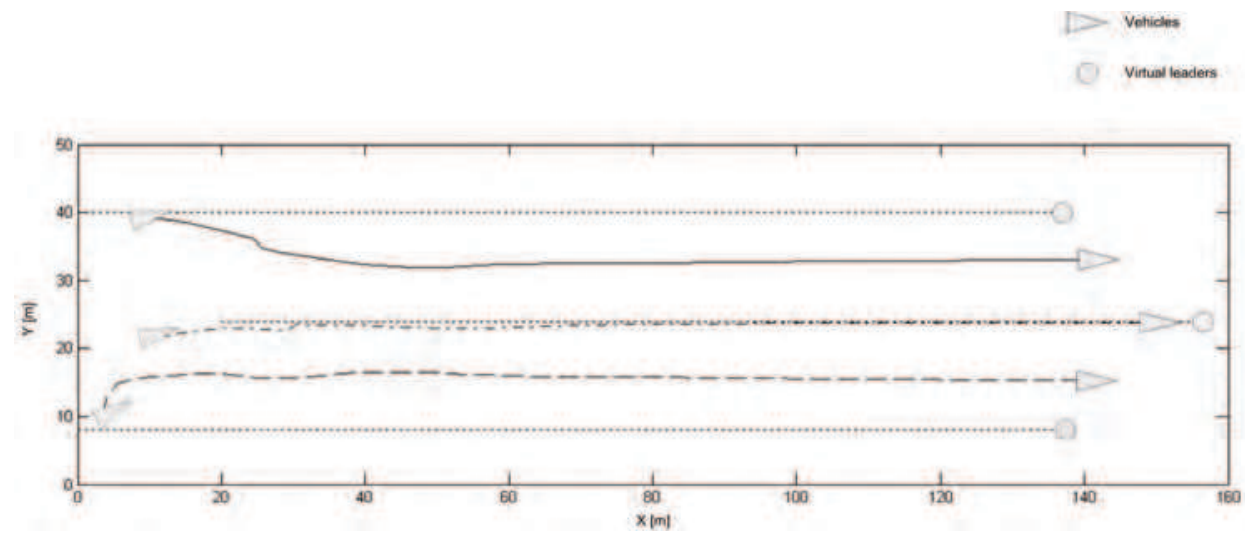

Fig. 7. Schooling of the vehicles in a triangular movement.

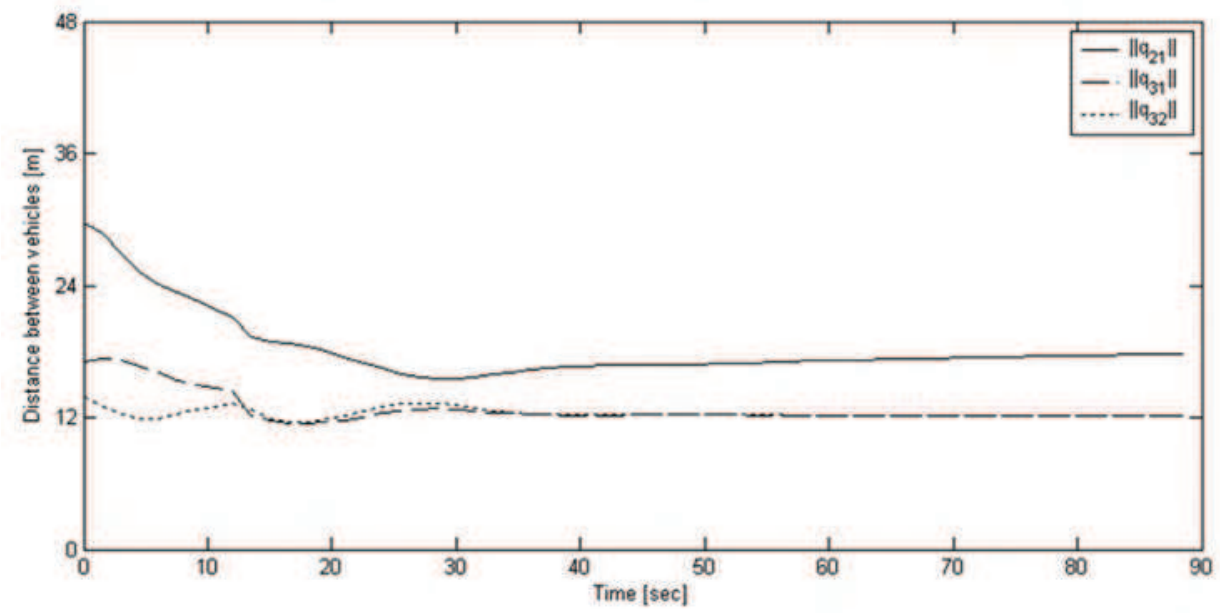

Fig. 8. Schooling geometry for a triangular movement.

\subsection{Equilateral triangular schooling with obstacle avoidance}

In this case, we consider an equilateral triangular schooling of the vehicles with obstacle avoidance. The obstacle is modelled as a circle located at $q_{p}=(40,10)$ with radius as $3 \mathrm{~m}$. For 
the schooling, two virtual leaders are chosen as in Fig. 3 (c) with the initial positions taken as $q_{v 1}(0)=(2 \sqrt{3}, 10)$ and $q_{v 2}(0)=(10 \sqrt{3}, 10)$. The vehicles' initial conditions are taekn as $q_{1}(0)=$ $(0,1), q_{2}(0)=(2,15), q_{3}(0)=(7 \sqrt{3}, 11), \psi_{1}(0)=0.1 \mathrm{rad}, \psi_{2}(0)=0.05 \mathrm{rad}, \psi_{3}(0)=0.15 \mathrm{rad}$ and $u_{1}(0)$ $=u_{2}(0)=u_{3}(0)=0.5 \mathrm{~m} / \mathrm{s}$ with all other variables taking zero values. Other design parameters are taken as $k_{u i}=18, k_{\psi i}=10, k_{r i}=12, i=1, \cdots, 3$ and $\gamma_{\alpha}=0.15, \gamma_{\beta}=0.2, \gamma_{\gamma}=0.15, \gamma_{u}=240$, $\gamma_{\psi}=6$.

Both $V_{\alpha}$ and $V_{\beta}$ are also taken as the form as (6) with the parameters as $a_{\alpha}=12, b_{\alpha}=30$, $a_{\beta}=4 \sqrt{3}, b_{\beta}=30, a_{\gamma}=b_{\gamma}=6, c_{\alpha}=4, c_{\beta}=5, c_{\gamma}=90$ and $h=0.9$.

Simulation results are depicted in Fig. 9 12. Fig. 9 shows the vehicles schooling in the equilateral triangular movement with obstacle avoidance. From Fig. 10, we can see that there is not any collision between vehicles. Fig. 11 presents the vehicles' velocity and heading matching in the schooling, and Fig. 12 shows the histories of proposed formation control laws for $\tau_{u i}$ and $\delta_{r i}$.

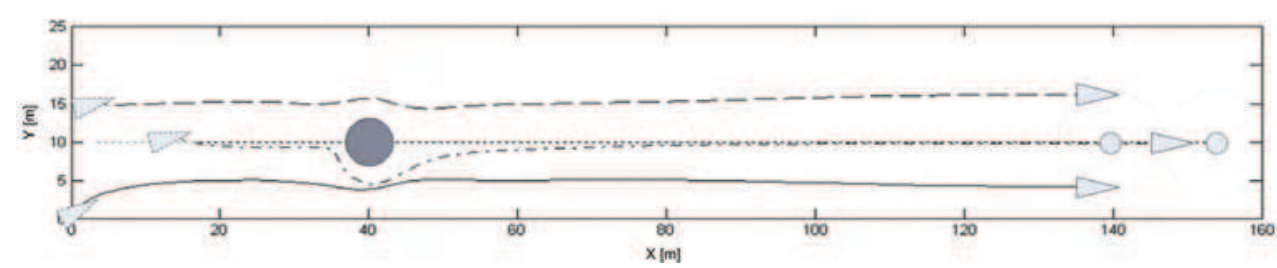

Fig. 9. Schooling of the vehicles in an equilateral triangular movement with obstacle avoidance.

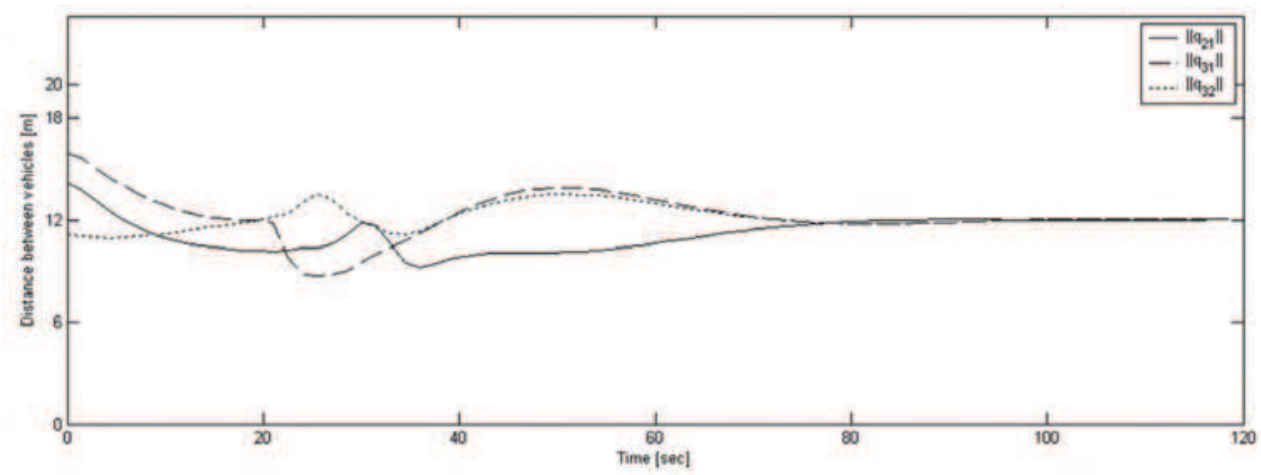

Fig. 10. Schooling geometry for an equilateral triangular movement with obstacle avoidance. 

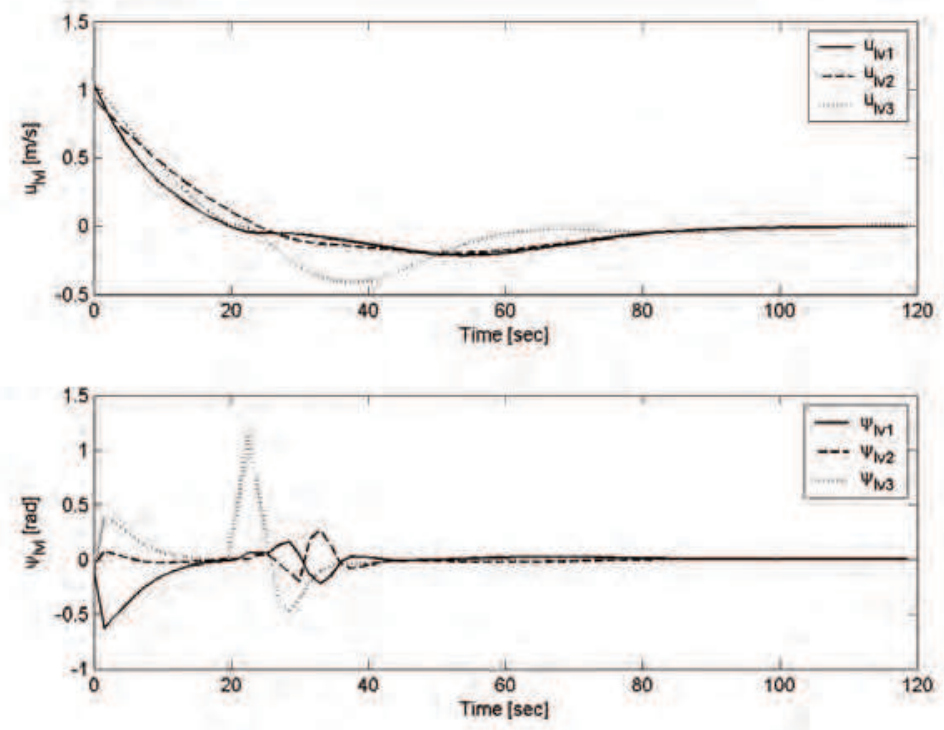

Fig. 11. Group velocity and heading matching.
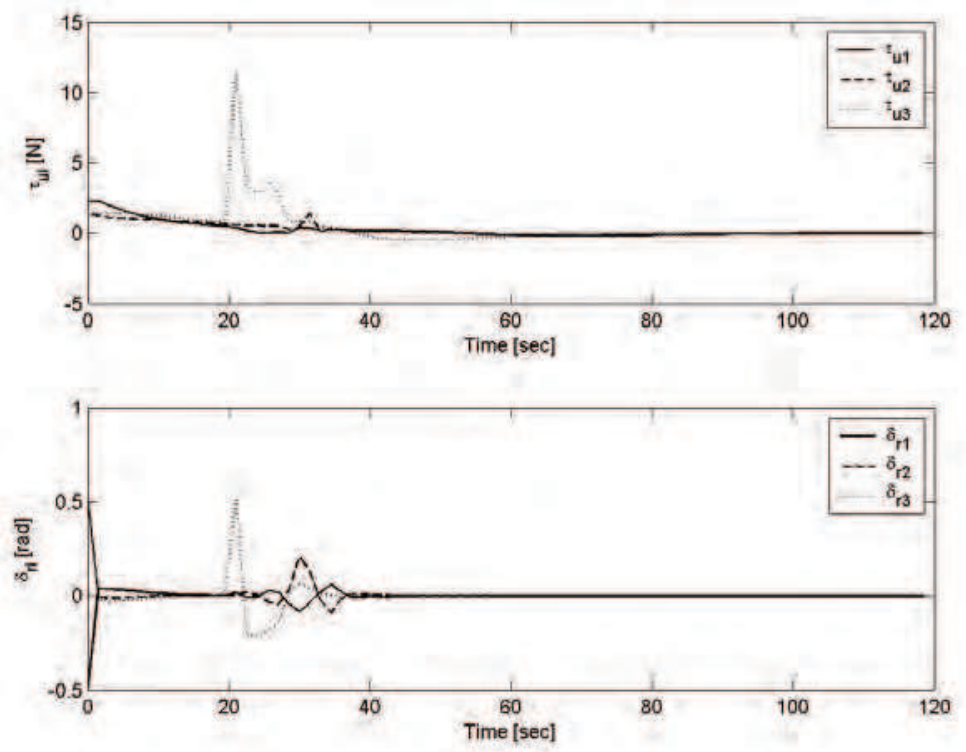

Fig. 12. Histories of proposed formation control laws. 


\section{Summary}

In this chapter, we have investigated an asymptotic schooling scheme for multiple underactuated underwater vehicles. For each vehicle, there are only two control inputs surge force and yaw moment available for its three DOF motion in the horizontal plane. The main difficulty in the tracking of this kind of vehicle is how to properly handle the vehicle's sway dynamics. To deal with this problem, in this chapter, we have introduced a certain polar coordinates transformation, through which the vehicle's dynamics can be reduced to a two-inputs strict-feedback form. The vehicles schooling has been conducted by properly selected smooth potential function, which consists of three different parts: one is for the interaction between vehicles, another is for group navigation, and the third one is for obstacle avoidance. The proposed formation algorithm guarantees the vehicles asymptotic schooling and velocity and heading matching while keeping obstacle avoidance.

Proposed schooling scheme has been derived under the condition of $u(t) \geq u_{\min }>0$, which inversely can be guaranteed by proposed formation control laws being combined with some suitable initial conditions. Therefore, the proposed schooling method only can guarantee the local stability. Moreover, it is notable that the following issues should be considered in our future works.

- Finite cut-off ( $b<+\infty$ in Definition 1) of potential function, which was applied in the previous works (Leonard and Fiorelli, 2001; Olfati-Saber, 2006; Do, 2007), also plays an important role in the vehicles schooling in this chapter. However, since $b<+\infty$, it is easy to verify that $\partial f_{p}(\zeta, a, b) / \partial \zeta=0$ if $\zeta \geq b$. For this reason, the proposed schooling scheme only guarantees certain local minimum. It is of interest to upgrade the present result to the one where the global minimum can be guaranteed in our future works.

- Another practical concern is for the robustness of proposed schooling scheme. In practice, there various uncertainty terms have to be faced, such as vehicle's modelling error, measurement noise, and disturbance, etc. All of these terms should be considered in our future practical applications.

\section{Acknowledgements}

This work was supported by the Ministry of Land, Transport and Maritime Affairs in Korea under Grant PMS162A and by the Korea Ocean Research \& Development Institute under Grant PES120B.

\section{References}

Bacciotti. A. \& Rosier, L. (2005). Liapunov Functions and Stability in Control Theory. SpringerVerlag, Berlin, Heidelberg, 2005

Brockett, R. W. ; Mullman, R. S. \& Sussmann, H. J. (1983). Differential Geometric Control Theory. Boston, MA : Birkhauser, 1983

Do, K. D. (2007). Bounded controller for formation stabilization of mobile agents with limited sensing ranges. IEEE Transactions on Automatic Control, Vol. 52, No. 2, pp. 569-576

Do, K. D. \& Pan, J. (2005). Global tracking control of underactuated ships with nonzero offdiagonal terms in their system matrices. Automatica, Vol. 41, No. 1, pp. 87-95 
Do, K. D. ; Jiang, Z. P. \& Pan, J. (2002a). Underactuated ship global tracking under relaxed conditions. IEEE Transactions on Automatic Control, Vol. 47, No. 9, pp. 1529-1536

Do, K. D. ; Jiang, Z. P. \& Pan, J. (2002b). Universal controllers for stabilization and tracking of underactuated ships. Systems \& Control Letters, Vol. 47, No. 4, pp. 299-317

Do, K. D. ; Jiang, Z. P. \& Pan, J. (2004). Robust adaptive path following of underactuated ships. Automatica, Vol. 40, Nol 6, pp. 929-944

Dunbar, W. B. \& Murray, R. M. (2002). Model predictive control of coordinated multivehicle formations, Proceedings of the 41st IEEE Conference on Decision and Control, pp. 4631-4636, Las Vegas, Nevada, USA, December 2002

Edwards, D. D. ; Bean, T. A.; Odell, D. L. \& Anderson, M. J. (2004). A leader-follower algorithm for multiple AUV formations, Proceedings of Workshop on Autonomous Underwater Vehicles, 2004 IEEE/OES, pp. 40-46, Sebasco Estates, Maine, USA, June 2004

Fax, J. A. \& Murray, R. M. (2004). Information flow and cooperative control of vehicle formations. IEEE Transaction on Automatic Control, Vol. 49, No. 9, pp. 1465-1476,

Fiorelli, E. ; Leonard, N. E. ; Bhatta, P. ; Paley, A. ; Bachmayer, R. \& Fratantoni, D. M. (2006). Multi-AUV control and adaptive sampling in Monterey Bay. IEEE Journal of Oceanic Engineering, Vol. 31, No., 4, pp. 935-948

Fossen, T. I. (2002). Marine Control Systems. Trondheim, Norway : Marine Cybermetics, 2002

Fredriksen, E. \& Pettersen, K. Y. (2006). Global k-exponential way-point maneuvering of ships : Theory and experiments. Automatica, Vol. 42, No. 4, pp. 677-687

Gue, J. ; Wei, Y. Y.; Chiu, F. C. \& Cheng, S. W. (2004). A maximum entropy method for multi-AUV grouping, Proceedings of IEEE/MTS Oceans'04, pp. 532-536, Kobe, Japan, November 2004

Jiang, Z. P. (2002). Global tracking control of underactuated ships by Lyapunov's direct method. Automatica, Vol. 38, No. 2, pp. 301-309

Jiang, Z. P. \& Nijmeijer, H. (1999). A recursive technique for tracking control of nonholonomic systems in chained form. IEEE Transactions on Automatic Control, Vol. 44, No. 2, pp. 265-279

Krstic, M. ; Kanellakopoulos, I. \& Kokotovic, P. (1995). Nonlinear and Adaptive Control Design. John Wiley \& Sons, Inc., New York, 1995

Latombe, J. (1991). Robot Motion Planning. Norwell, MA :Kluwer, 1991

Lee, P. M. et al. (2003). Development of an Advanced Deep-Sea Unmanned Underwater Vehicle (II). Technical Report, UCM0043A-2442, KORDI, Daejeon, Korea, 2003

Leonard, N. E. \& Fiorelli, E. (2001). Virtual leaders, artificial potentials and coordinated control of groups, Proceedings of the 40th IEEE Conference on Decision and Control, pp. 2968-2973, Orlando, Florida, USA, December 2001

Li, J. H. \& Lee, P. M. (2008). Stable schooling for multiple underactuated AUVs, Proceedings of IFAC World Congress, pp. 15022-15027, Seoul, Korea, July 2008

Marthiniussen, R. ; Vestgard, K. ; Klepaker, R. A. \& Storkersen, N. (2004). HUGIN-AUV concept and operational experience to date, Proceedings of IEEE/MTS Oceans'04, pp. 846-850, Kobe, Japan, November 2004

Murray, R. M. \& Sastry, S. S. (1993). Nonholonomic motion planning: Steering using sinusoids. IEEE Transactions on Automatic Control, Vol. 38, No. 5, pp. 700-716

Newman, J. N. (1977). Marine Hydrodynamics. The MIT Press, Cambridge, Massachusetts, USA, and London, England, 1977 
Olfati-Saber, R. (2006). Flocking for multi-agent dynamic systems : algorithms and theory. IEEE Transactions on Automatic Control, Vol. 51, No. 3, pp. 401-420

Olfati-Saber, R. \& Murray, R. M. (2002). Distributed cooperative control of multiple vehicle formations using structural potential functions, Proceedings of 15th IFAC World Congress, Barcelona, Spain, July 2002

Olfati-Saber, R. \& Murray, R. M. (2003). Flocking with obstacle avoidance : cooperation with limited communication in mobile networks, Proceedings of the 42nd IEEE Conference on Decision and Control, pp. 2022-2028, Maui, Hawaii, USA, December 2003

Pettersen, K. Y. \& Nijmeijer, H. (2001). Underactuated ship tracking control : Theory and experiments. International Journal of Control, Vol. 74, No. 14, pp. 1435-1446

Prestero, T. (2001). Verificatin of a six-degree of freedom simulation model for the REMUS autonomous underwater vehicles. Masters Thesis, Department of Ocean Engineering and Mechanical Engineering, MIT, 2001

Rey nolds, C. W. (1987). Flocks, herds, and schools: A distributed behavioral model, Proceedings of ACM SIGGRAPH'87, pp. 25-34, Anaheim, California, USA, July 1987

Reyhanoglu, M. (1997). Exponential stabilization of an underactuated autonomous surface vessel. Automatica, Vol. 33, No. 12, pp. 2249-2254

Rimon, E. \& Koditschek, D. E. (1992). Exact robot navigation using artificial potential function. IEEE Transactions on Robotics and Automation, Vol. 8, No. 5, pp. 501-518

Smith, T. R. ; Hanbmann, H. \& Leonard, N. E. (2001). Orientation control of multiple underwater vehicles with symmetry-breaking potentials, Proceedings of the 40th IEEE Conference on Decision and Control, pp. 4598-4603, Orlando, Florida, USA, December 2001

Sousa, J. B. \& Pereira, F. L. (2002). Coordinated control strategies for networked vehicles : an application to autonomous underwater vehicles, Proceedings of International Workshop on Generalized Solutions in Control Problems, Perslavl-Zalessky, Russia, July 2002

Watanabe, K. \& Nakamura, A. (2005). A design of tiny basin test-bed for AUV multi agent, Proceedings of IEEE/MTS Oceans 2005, pp. 1002-1008, Washington D. C., USA, September 2005

Westwood, R.; Westwood, J. \& Newman, P. (2007). The AUV Gamechanger Report 2008-2017. Douglas-Westwood Limited, Canterbury, UK, 2007 


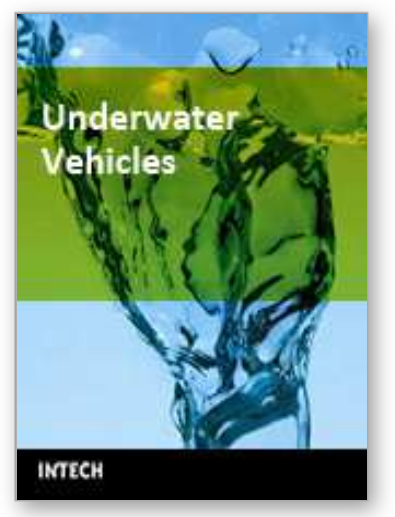

\author{
Underwater Vehicles \\ Edited by Alexander V. Inzartsev
}

ISBN 978-953-7619-49-7

Hard cover, 582 pages

Publisher InTech

Published online 01, January, 2009

Published in print edition January, 2009

For the latest twenty to thirty years, a significant number of AUVs has been created for the solving of wide spectrum of scientific and applied tasks of ocean development and research. For the short time period the AUVs have shown the efficiency at performance of complex search and inspection works and opened a number of new important applications. Initially the information about AUVs had mainly review-advertising character but now more attention is paid to practical achievements, problems and systems technologies. AUVs are losing their prototype status and have become a fully operational, reliable and effective tool and modern multi-purpose AUVs represent the new class of underwater robotic objects with inherent tasks and practical applications, particular features of technology, systems structure and functional properties.

\title{
How to reference
}

In order to correctly reference this scholarly work, feel free to copy and paste the following:

Ji-Hong Li and Pan-Mook Lee (2009). Schooling for Multiple Underactuated AUVs, Underwater Vehicles, Alexander V. Inzartsev (Ed.), ISBN: 978-953-7619-49-7, InTech, Available from:

http://www.intechopen.com/books/underwater_vehicles/schooling_for_multiple_underactuated_auvs

\section{INTECH}

open science | open minds

\section{InTech Europe}

University Campus STeP Ri Slavka Krautzeka 83/A 51000 Rijeka, Croatia Phone: +385 (51) 770447

Fax: +385 (51) 686166 www.intechopen.com

\section{InTech China}

Unit 405, Office Block, Hotel Equatorial Shanghai No.65, Yan An Road (West), Shanghai, 200040, China 中国上海市延安西路65号上海国际贵都大饭店办公楼405单元 Phone: +86-21-62489820

Fax: +86-21-62489821 
(C) 2009 The Author(s). Licensee IntechOpen. This chapter is distributed under the terms of the Creative Commons Attribution-NonCommercialShareAlike-3.0 License, which permits use, distribution and reproduction for non-commercial purposes, provided the original is properly cited and derivative works building on this content are distributed under the same license. 